\title{
Protecção das Pessoas em Instalações Eléctricas de Baixa Tensão Cálculo dos Dispositivos de Protecção
}

RESUMO

O correcto dimensionamento dos dispositivos de protecção das pessoas contra contactos indirectos em instalações eléctricas de Baixa Tensão (BT), é uma das condições fundamentais para que uma instalação possa ser utilizada e explorada com conforto e em perfeitas condições de segurança. De acordo com a normalização em vigor, é, também, uma das condições essenciais para a certificação ou licenciamento das instalações eléctricas por parte das entidades ou organismos responsáveis, a quem estão atribuídas estas competências.

A função dos dispositivos de protecção das pessoas contra os contactos indirectos será o corte automático da alimentação da instalação eléctrica, que, em caso de defeito, e em consequência do valor e da duração da tensão de contacto, evitará o risco de se produzirem efeitos fisiopatológicos perigosos nas pessoas. Esta medida de protecção obriga à coordenação entre o Regime de Neutro lou Esquema de Ligação à Terra (ELT)) adoptado na instalação, e as características dos condutores de protecção e dos respectivos dispositivos de proteç̧ão.

Neste artigo são apresentados alguns exemplos de cálculo dos dispositivos de protecção das pessoas contra contactos indirectos, de acordo com o Regime de Neutro adoptado para a instalação eléctrica.

\section{CÁLCULOS NO REGIME DE NEUTRO “TN”}

Este regime de neutro caracteriza-se por todas as massas da instalação serem ligadas ao ponto da alimentação ligado à terra, próximo do transformador ou do gerador da alimentação da instalação, por meio de condutores de protecção.

O ponto da alimentação ligado à terra é, em regra, o ponto neutro.

De acordo com a legislação em vigor, nas instalações fixas pode-se utilizar um só condutor com as funções de condutor de protecção e de condutor neutro (designado por condutor PEN) desde que o condutor de protecção tenha uma secção não inferior a $10 \mathrm{~mm}^{2}$, se de cobre ou a $16 \mathrm{~mm}^{2}$, se de alumínio e, a parte da instalação comum (esquema TN-C) não esteja localizada a jusante de um dispositivo diferencial.

Este regime de neutro encontra-se representado na Figura 1.

Neste regime de neutro um defeito de isolamento é similar a um curto-circuito entre fase e neutro, e o corte deve ser assegurado pelo dispositivo de protecção contra curtoscircuitos, com um tempo máximo de corte especificado que é função da tensão limite convencional (UL) admissível para o local da instalação, ou seja, 25V ou 50V em corrente alternada, sendo o valor definido pela classificação do local quanto às influências externas.

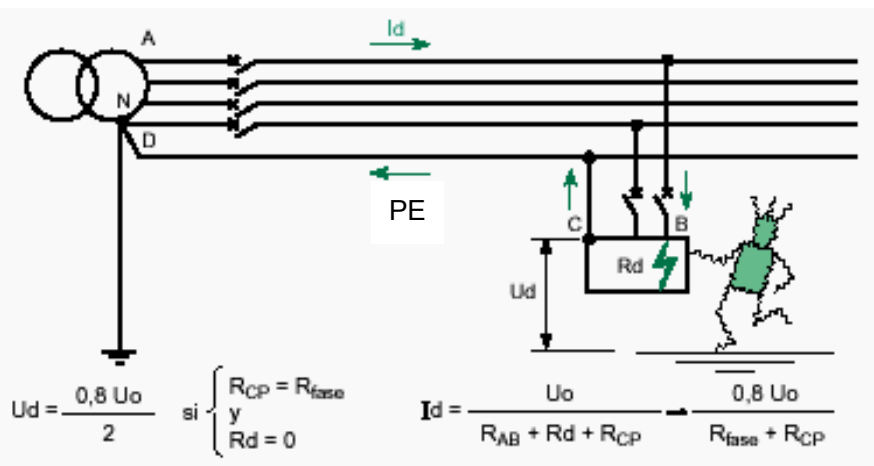

Figura 1: Regime terra pelo neutro, ou esquema TN (Fonte Schneider Electric) 


\section{ARTIGO TÉCNICO}

Segundo a norma CEI 364 o tempo de corte do dispositivo de protecção deverá ser de $0,4 \mathrm{~s}$ para $\mathrm{UL}=50 \mathrm{~V}$ e, $0,2 \mathrm{~s}$ para $U L=25 \mathrm{~V}$.

Seguidamente, apresenta-se um circuito de uma instalação eléctrica de BT, trifásica (400V), onde é adoptado o regime de neutro TN-C, ou seja, a função de neutro e de protecção estão combinadas num único condutor (PEN).

Este circuito é apresentado na figura 2.

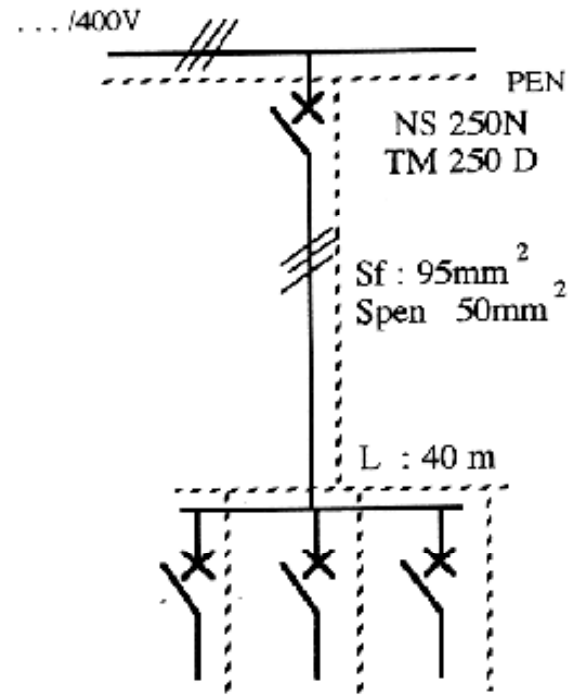

Figura 2: Exemplo de cálculo. Regime TN-C

O circuito tem um comprimento de $40 \mathrm{~m}$, a secção do condutor de fase é de $95 \mathrm{~mm}^{2}$ e a do condutor de protecção é de $50 \mathrm{~mm}^{2}$.

O circuito está protegido com disjuntor NS 250N (Merlin Gerin) equipado com disparador magnetotérmico TM 250 curva D.

Pretende-se verificar se neste regime de neutro, a protecção das pessoas contra contactos indirectos está efectivamente garantida com este dispositivo de protecção.

Uma condição fundamental para o correcto dimensionamento do dispositivo de protecção, é conhecer a sua curva de actuação, de maneira a obter-se o valor da corrente correspondente ao limiar de funcionamento do disparador magnético do aparelho de protecção.
A curva deste dispositivo de protecção é apresentada na figura 3.

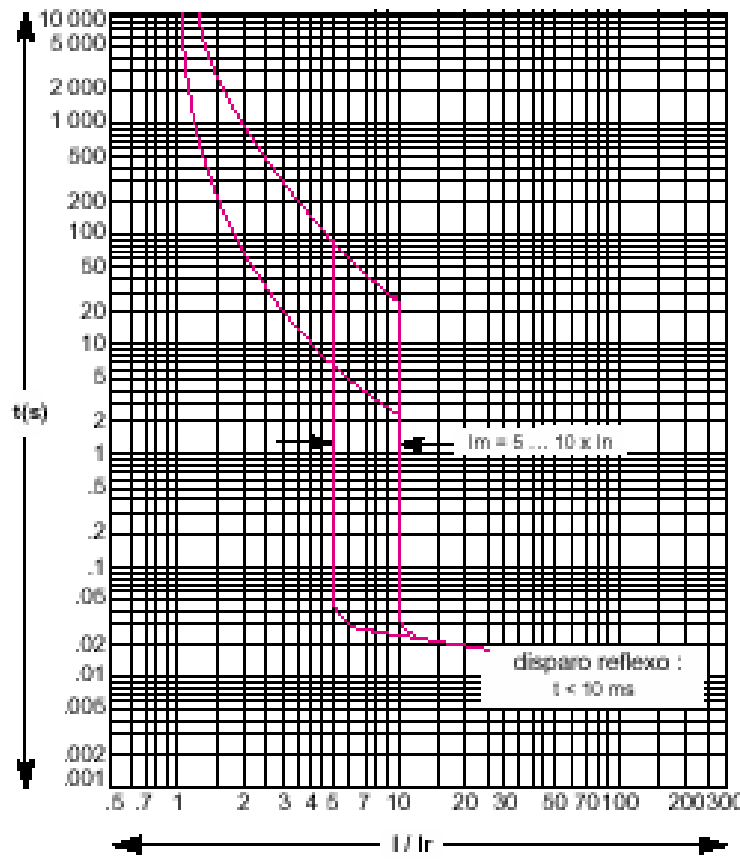

Figura 3: Curva de disparo TM250D.

(Fonte Schneider Electric)

Como se pode verificar, a actuação do disparador magnético deste disjuntor poderá ser regulada para funcionar entre 5 a 10 vezes o valor nominal (In), ou seja, entre 1250 e $2500 \mathrm{~A}$.

Neste regime de neutro a impedância da malha de defeito Zs será:

$$
Z_{s}=\frac{K \cdot U_{0}}{I_{d}}
$$

em que $\mathrm{K}$ toma o valor de 0,8 para instalações eléctricas, $U_{0}$ é a tensão simples nominal da instalação e $I_{d}$ é a corrente de defeito.

Para que a protecção contra curtos-circuitos também garanta a protecção contra contactos indirectos, é necessário para os disjuntores que:

$$
Z_{s} \leq \frac{K \cdot U_{0}}{I_{m}}
$$

em que $I_{m}$ é a corrente de actuação do disparador magnético do dispositivo. 


\section{ARTIGO TÉCNICO}

Para a protecção por fusíveis, é necessário que:

$$
Z_{s} \leq \frac{K \cdot U_{0}}{I_{f}}
$$

em que $I_{f}$ é a corrente convencional de funcionamento do fusível.

Atendendo a que neste regime de neutro um defeito é efectivamente um curto-circuito entre uma fase e o condutor de protecção, a impedância da malha de defeito será então:

$$
Z_{s} \approx R_{s}=\rho_{f} \frac{l}{s_{f}}+\rho_{P E} \frac{l}{s_{P E}}
$$

em que $\rho_{\mathrm{f}}$ é a resistividade de condutor de fase, $\rho_{\mathrm{PE}}$ a resistividade do condutor de protecção, I é o comprimentos dos condutores, $s_{f}$ a secção do condutor de fase e $s_{P E} a$ secção do condutor de protecção.

Considerando que os condutores de fase e de protecção têm as mesmas características, a impedância da malha de defeito será então:

$$
Z_{s} \approx R_{s}=\rho \frac{l}{s_{f}} .(1+m)
$$

em que ;

$$
m=\frac{s_{f}}{S_{P E}}
$$

O comprimento máximo protegido do circuito será então, para disjuntores:

$$
l \leq \frac{K \cdot U_{0} \cdot S_{f}}{\rho \cdot(1+m) \cdot I_{m}}
$$

e para fusíveis será de:

$$
l \leq \frac{K \cdot U_{0} \cdot s_{f}}{\rho \cdot(1+m) \cdot I_{f}}
$$

Para o circuito apresentado na figura 2, o comprimento máximo protegido do circuito, para uma regulação do disparador magnético de 5xIn (Im=1250A) será de:

$$
l \leq \frac{0,8 \cdot 230 \cdot 95}{0,0225 \cdot(1+19) \cdot 1250} \leq 214 m
$$

para uma regulação do disparador magnético de 10xIn (Im=2500A) será de:

$$
l \leq \frac{0,8 \cdot 230 \cdot 95}{0,0225 \cdot(1+19) \cdot 2500} \leq 107 m
$$

Atendendo que o comprimento do circuito é de $40 \mathrm{~m}$, verifica-se que em qualquer dos casos o disjuntor garante a protecção das pessoas contra contactos indirectos.

No entanto, deve-se também verificar se o tempo de actuação do dispositivo é compatível com o especificado pelas curvas de segurança, para a tensão limite convencional definida para o local da instalação, que como já foi referido, segundo a norma CEI 364 deverá ser de $0,4 \mathrm{~s}$ para $\mathrm{U}_{\mathrm{L}}=50 \mathrm{~V}$ e, $0,2 \mathrm{~s}$ para $\mathrm{U}_{\mathrm{L}}=25 \mathrm{~V}$.

Assim, torna-se importante calcular o valor da tensão de contacto em caso de defeito.

$$
U_{c}=R_{P E} \cdot I_{d}
$$

em que:

$$
I_{d}=\frac{K \cdot U_{0}}{Z_{s}}=\frac{K \cdot U_{0}}{\rho \cdot \frac{i}{s_{f}} \cdot(1+m)}
$$

então:

$$
U_{c}=R_{P E} \cdot \frac{K \cdot U_{0} \cdot S_{f}}{\rho \cdot l \cdot(1+m)}
$$

$$
\begin{aligned}
& R_{P E}=\rho \frac{l}{S_{P E}} \\
& U_{c}=K \cdot U_{0} \cdot \frac{m}{1+m}
\end{aligned}
$$


Para o exemplo em consideração, representado na figura 2, tem-se:

$$
U_{c}=0,8 * 230 * \frac{1,9}{1+1,9}=120,6 \mathrm{~V}
$$

Pelas curvas de segurança, e para a tensão limite convencional de $25 \mathrm{~V}$, o dispositivo deve actuar num tempo inferior a $180 \mathrm{~ms}$.

Como se pode verificar na curva de funcionamento do disjuntor, apresentada na figura 3 , o dispositivo actuará num tempo inferior ao referido e compatível com o especificado pela norma CEI 364.

Assim, para esta instalação, e para este regime de neutro, pode-se garantir que o disjuntor apresentado protege efectivamente as pessoas contra contactos indirectos.

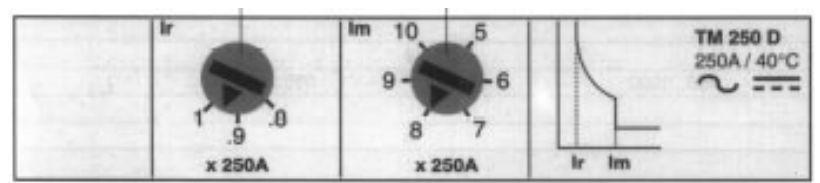

Figura 4: Painel de regulação do relé electrónico TM250D.

(Fonte Schneider Electric)

\section{CÁlCULOS NO REGIME DE NEUTRO “IT"}

Este esquema de ligação à terra apresenta como principal vantagem, a garantia de continuidade de serviço em presença de um primeiro defeito de isolamento.
Neste regime de neutro, a presença de um primeiro defeito não origina valores de tensão de contacto perigosos para as pessoas.

No entanto, é obrigatório a presença de um Controlador Permanente de Isolamento (CPI), de maneira a sinalizar o defeito e permitir a sua eliminação o mais rapidamente possível.

A manifestação de um segundo defeito, sem que tenha sido eliminado o primeiro, implicaria agora a existência de tensões de contacto muito perigosas, devendo ser tomadas as medidas adequadas de forma a evitar riscos de efeitos fisiopatológicos perigosos nas pessoas susceptíveis de ficar em contacto com partes condutoras simultaneamente acessíveis.

Como tal, a protecção das pessoas neste regime de neutro é orientada para o dimensionamento dos dispositivos de protecção actuarem na situação de segundo defeito.

Também se devem eliminar todas as situações que possam contribuir para diminuir a fiabilidade do sistema. Assim, não se deve distribuir o condutor neutro, pois poderá correr-se o risco de manifestar-se um segundo defeito sem que o primeiro tenha sido sinalizado, actuando a proteç̧ão e perdendo-se todas as vantagens inerentes à utilização deste regime de neutro.

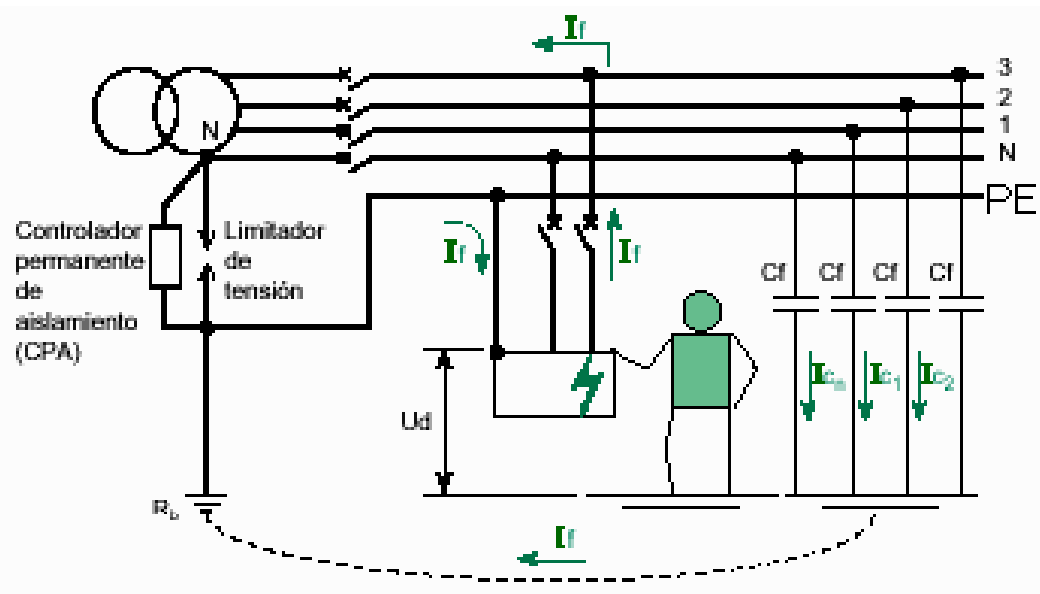

Figura 5: Esquema de Ligação à Terra IT. (Fonte Schneider Electric) 


\section{ARTIGO TÉCNICO}

Este regime de neutro caracteriza-se por as partes activas da instalação eléctrica serem isoladas da terra ou ligadas a esta através de uma impedância de valor elevado. As massas dos aparelhos de utilização são ligadas à terra, individualmente ou por grupos.

A situação mais comum nas instalações onde é adoptado este regime de neutro, é todas as massas, incluindo as da fonte, estarem ligadas a um mesmo eléctrodo de terra (figura 5). Assim, as condições de eliminação da corrente de um segundo defeito são então garantidas pelas mesmas condições indicadas para o esquema TN.

Neste regime de neutro IT, a protecção das pessoas contra contactos indirectos é fundamentalmente garantida por dois tipos de equipamentos:

- pelos $\mathrm{CPI}$, essencialmente destinados à vigilância do primeiro defeito, embora possam também ser utilizados como dispositivos de protecção nas situações em que for necessário provocar o corte ao primeiro defeito;

- pelos dispositivos de protecção contra sobreintensidades (disjuntores e fusíveis). Estes dispositivos são utilizados nas situações em que ao segundo defeito são aplicadas as condições de protecção definidas para o esquema TN;

Seguidamente, apresenta-se um circuito de uma instalação eléctrica de BT, trifásica (400V), onde é adoptado o regime de neutro IT, sem neutro distribuído (situação comum neste regime de neutro) . Este circuito é apresentado na figura 6 .

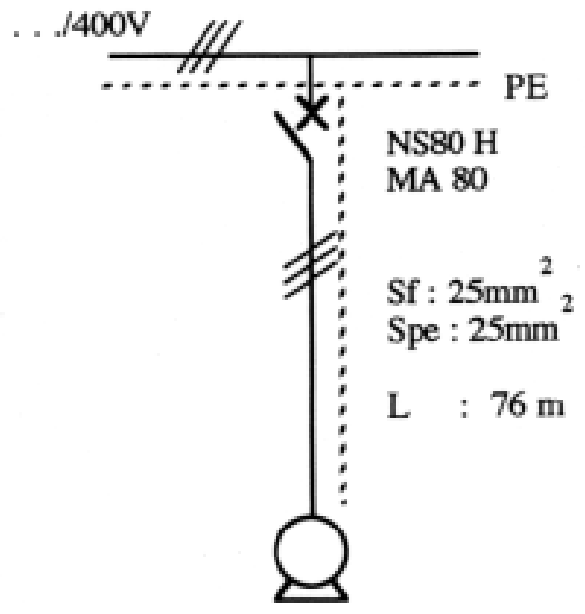

Figura 6: Exemplo de cálculo. Regime IT
O circuito tem um comprimento de $76 \mathrm{~m}$, a secção do condutor de fase e de protecção é de $25 \mathrm{~mm}^{2}$. O circuito está protegido com disjuntor especifico para protecção de saídas motor NS $80 \mathrm{H}$ (Merlin Gerin) equipado com disparador "motor" integrado MA 80.

Pretende-se verificar se neste regime de neutro, a protecção das pessoas contra contactos indirectos está efectivamente garantida com este dispositivo de protecção.

Também no caso deste regime de neutro é fundamental para o correcto dimensionamento do dispositivo de protecção, conhecer a curva de actuação do dispositivo, de maneira a obter-se o valor da corrente correspondente ao limiar de funcionamento do disparador magnético do aparelho de protecção.

A curva deste dispositivo de protecção é apresentada na figura 7.

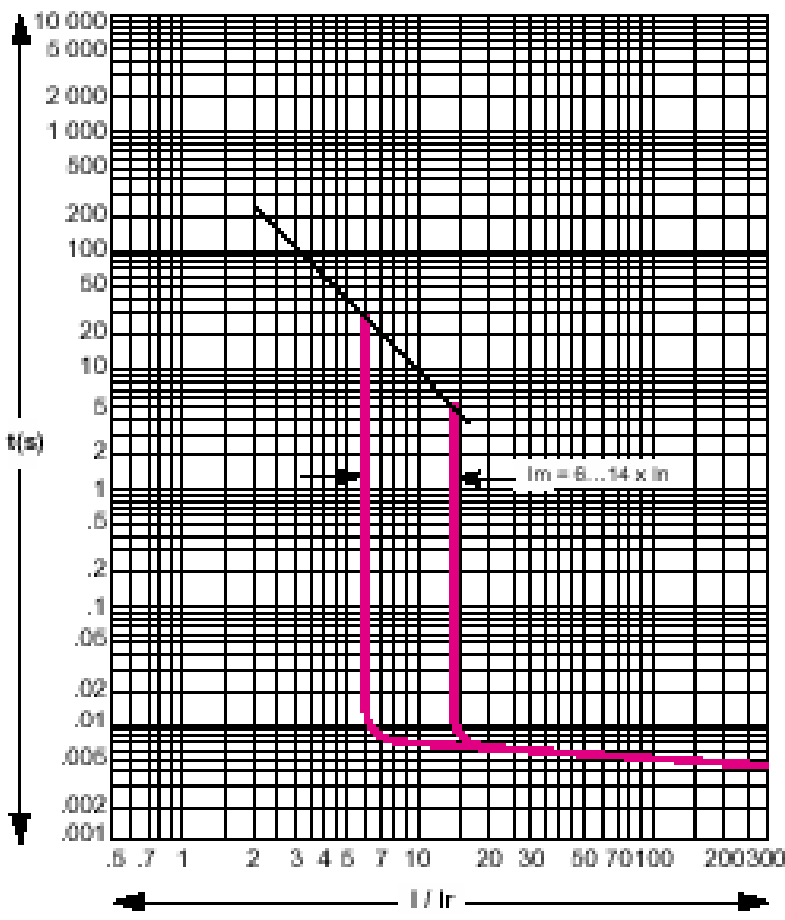

Figura 7: Curva de disparo MA80.

(Fonte Schneider Electric)

Como se pode verificar, a actuação do disparador magnético deste disjuntor verifica-se entre 6 a 14 vezes o valor nominal (In=80A), ou seja, entre 480 e 1120 A. 
Também neste regime de neutro, tal como no regime TN, um defeito é efectivamente um curto-circuito entre uma fase e o condutor de protecção.

Então, para este circuito, sem neutro distribuído, a impedância da malha de defeito será:

$$
Z_{s} \leq \frac{K \cdot \sqrt{3} \cdot U_{0}}{I_{m}}
$$

em que $I_{m}$ é a corrente de actuação do disparador magnético do dispositivo.

Neste regime de neutro considera-se como boa aproximação que ao segundo defeito, o comprimento da malha de defeito é duplo em relação ao primeiro defeito.

Então, a impedância da malha de defeito será neste caso:

$$
Z_{s} \approx R_{s}=2 *\left(\rho_{f} \frac{l}{s_{f}}+\rho_{P E} \frac{l}{S_{P E}}\right)
$$

Considerando também que os condutores de fase e de protecção têm as mesmas características, a impedância da malha de defeito será então:

$$
Z_{s} \approx R_{s}=2 *\left(\rho \frac{l}{s_{f}} \cdot(1+m)\right)
$$

em que ;

$$
m=\frac{s_{f}}{S_{P E}}=1
$$

O comprimento máximo protegido deste circuito será então, para disjuntores:

$$
l \leq \frac{K \cdot \sqrt{3} \cdot U_{0} \cdot S_{f}}{2 \cdot \rho \cdot(1+m) \cdot I_{m}}
$$

Para o circuito apresentado na figura 6, o comprimento máximo protegido do circuito, para uma regulação do disparador magnético de $6 x \ln (\operatorname{Im}=480 A)$ será de:

$$
l \leq \frac{0,8 \cdot \sqrt{3} \cdot 230 \cdot 25}{2 \cdot 0,0225 \cdot(1+1) \cdot 480} \leq 184 m
$$

Para uma regulação do disparador magnético de 14xln $(\mathrm{Im}=1120 A)$ será de:

$$
l \leq \frac{0,8 \cdot \sqrt{3} \cdot 230 \cdot 25}{2 \cdot 0,0225 \cdot(1+1) \cdot 1120} \leq 79 m
$$

Atendendo que o comprimento do circuito é de $76 \mathrm{~m}$, verifica-se que para qualquer regulação do disparador MA (6 a $14 x \ln$ ), o disjuntor garante a protecção das pessoas contra contactos indirectos.

No entanto, tal como no regime de neutro TN, também se deve verificar se o tempo de actuação do dispositivo é compatível com o especificado pelas curvas de segurança, para a tensão limite convencional definida para o local da instalação, que como já foi referido, segundo a norma CEI 364 deverá ser de $0,4 \mathrm{~s}$ para $U_{\mathrm{L}}=50 \mathrm{~V}$ e, $0,2 \mathrm{~s}$ para $\mathrm{U}_{\mathrm{L}}=25 \mathrm{~V}$.

Assim, torna-se importante calcular o valor da tensão de contacto em caso de segundo defeito.

$$
U_{c}=R_{P E} \cdot I_{d}
$$

em que, através de uma dedução idêntica à efectuada para o regime de neutro TN, obtêm-se:

$$
U_{c}=K \cdot \sqrt{3} \cdot U_{0} \cdot \frac{m}{2 \cdot(1+m)}
$$

Para o exemplo em consideração, representado na figura 6, tem-se:

$$
U_{c}=0,8 * \sqrt{3} * 230 * \frac{1}{2 *(1+1)}=79,7 \mathrm{~V}
$$

Pelas curvas de segurança, e para a tensão limite convencional de $25 \mathrm{~V}$, o dispositivo deve actuar num tempo inferior a $280 \mathrm{~ms}$.

Como se pode verificar na curva de funcionamento do disjuntor, apresentada na figura 7, o dispositivo actuará num tempo inferior ao referido e compatível com o especificado pela norma CEI 364 
Assim, também para esta instalação, e para este regime de neutro, pode-se garantir que o disjuntor apresentado protege efectivamente as pessoas contra contactos indirectos.

\section{CONCLUSÕES}

Neste artigo apresentou-se dois exemplos de cálculo e dimensionamento dos dispositivos de protecção das pessoas contra contactos indirectos. Um exemplo para o regime de neutro TN, e outro para o regime de neutro IT.

Atendendo a que nestes regimes de neutro, e para o caso dos exemplos apresentados, uma situação de defeito é sempre uma situação de curto-circuito entre um condutor activo e a massa do equipamento de utilização, ou seja, um curto-circuito entre um condutor activo e o condutor de protecção, são, normalmente, os dispositivos de protecção contra sobreintensidades que terão a função de também garantir a protecção das pessoas contra contactos indirectos.

Na realidade, nos exemplos que são apresentados, o que se teve que fazer foi verificar se realmente o dispositivo de protecção contra curtos-circuitos também verificava as condições necessárias à protecção das pessoas contra contactos indirectos.

Este facto foi analisado através da verificação do máximo comprimento protegido.

Efectivamente, nestes dois regimes de neutro, para se poder dimensionar correctamente os dispositivos de protecção, é fundamental conhecer bem as características do circuito, nomeadamente comprimento da instalação, tipo de condutores, trajecto dos cabos, secção dos condutores, etc. Outro factor importante, é verificar se o dispositivo actua num tempo compatível com especificado pelas normas de segurança. Este facto depende das condições do local da instalação eléctrica.

De acordo com estas condições, a legislação em vigor impõe como tensão de contacto limite, $25 \mathrm{~V}$ ou $50 \mathrm{~V}$. Assim, torna-se importante calcular o valor da tensão de contacto em caso de defeito e, através da curva de segurança dos $25 \mathrm{~V}$ ou $50 \mathrm{~V}$, conforme o caso, obter o tempo máximo de actuação do dispositivo para que a tensão de contacto nunca ultrapasse o valor da tensão limite convencional.

Este facto obriga, também, a conhecer muito bem as curvas de funcionamento dos dispositivos de protecção, para verificar se esta regra do tempo de actuação também é garantida. No caso dos disjuntores, a zona de funcionamento magnético dos disparadores é quase instantânea, não sendo a regra do tempo de actuação problemática para este tipo de equipamento de proteç̧ão.

O facto torna-se mais importante quando os dispositivos de protecção são fusíveis.

O regime de neutro $\mathrm{TT}$, para o dimensionamento dos dispositivos de protecção das pessoas contra contactos indirectos, não obriga necessariamente a conhecer todas as características da instalação.

A análise do dimensionamento dos dispositivos de protecção para o regime TT será efectuada num próximo artigo.

\section{Bibliografia}

[1] Regras Técnicas das Instalações Eléctricas de Baixa Tensão" (Decreto-Lei n.ㅇ 226/2005 de 28 de Dezembro)

[2] Técnicas e Tecnologias em Instalações Eléctricas" - L. M. Vilela Pinto - Edição Certiel

[3] Instalações Eléctricas de Baixa Tensão. A Concepção e o Projecto" - Aulas de IELBT, José Beleza Carvalho, ISEP

[4] Instalações Eléctricas Industriais" - João Mamede Filho - Editora LTC 5a Edição

[5] Esquemas de Ligação à Terra em BT (Regimes de Neutro)" Caderno Técnico no 172 - Bernard Lacroix e Roland Calvas. Edição: Schneider Electric 